\title{
A Novel Homotopy Perturbation Algorithm Using Laplace Transform for Conformable Partial Differential Equations
}

\author{
Sajad Iqbal $\mathbb{D}^{1},{ }^{1}$ Mohammed K. A. Kaabar $\mathbb{D}^{2,3}$ and Francisco Martínez ${ }^{4}$ \\ ${ }^{1}$ Department of Mathematics, Zhejiang University, Hangzhou 310027, China \\ ${ }^{2}$ Gofa Camp, Near Gofa Industrial College and German Adebabay, Nifas Silk-Lafto 26649, Addis Ababa, Ethiopia \\ ${ }^{3}$ Institute of Mathematical Sciences, Faculty of Science, University of Malaya, Kuala Lumpur 50603, Malaysia \\ ${ }^{4}$ Department of Applied Mathematics and Statistics, Technological University of Cartagena, Cartagena 30203, Spain
}

Correspondence should be addressed to Mohammed K. A. Kaabar; mohammed.kaabar@wsu.edu

Received 16 October 2021; Revised 22 November 2021; Accepted 26 November 2021; Published 14 December 2021

Academic Editor: Muhammad Shoaib Anwar

Copyright (C) 2021 Sajad Iqbal et al. This is an open access article distributed under the Creative Commons Attribution License, which permits unrestricted use, distribution, and reproduction in any medium, provided the original work is properly cited.

In this article, the approximate analytical solutions of four different types of conformable partial differential equations are investigated. First, the conformable Laplace transform homotopy perturbation method is reformulated. Then, the approximate analytical solution of four types of conformable partial differential equations is presented via the proposed technique. To check the accuracy of the proposed technique, the numerical and exact solutions are compared with each other. From this comparison, we conclude that the proposed technique is very efficient and easy to apply to various types of conformable partial differential equations.

\section{Introduction}

The initial idea of fractional derivative in history goes back to the late $17^{\text {th }}$ century, when the French mathematician L'Hopital concerned with the meaning of $\left(\mathrm{d}^{n} y / \mathrm{d} x^{n}\right)$, when $n=1 / 2$. Since then, various definitions of this concept have been formulated from two conceptions: one of a global nature and the other one of a local nature. The global formulation of the fractional derivative is linked to the appearance of the fractional calculus itself, and the Caputo and Riemann-Liouville definitions are the best known $[1,2]$.

Khalil et al. suggested recently an alternative fractional derivative of local type, named as conformable derivative [3] to solve some issues concerning the challenge of solving fractional differential equations (F-DEs) of nonlocal type. The physical and geometrical interpretations of conformable derivatives have been discussed in $[4,5]$, respectively.

In several research studies, the methods of homotopy perturbation and Adomian decomposition have been employed to solve various types of F-DEs [6, 7]. Furthermore, a new numerical technique that is relied on the homotopy perturbation method (HPM) and Laplace transform (LT) has been proposed in [8] to solve F-DEs.
Madani et al. employed the Laplace transform homotopy perturbation method (LTHPM) to solve one-dimensional in-homogeneous partial differential equations (PDEs) with a variable coefficient [9]. In [10], LTHPM is discussed to obtain the approximate analytical solution of space-fractional and time-fractional Burgers equations.

Fall et al. [11] implemented the homotopy perturbation method to obtain the analytical solution of time-fractional Black-Scholes (T-BSEs) and the generalized fractional BSEs. They have displayed both the solution graphically and discussed the effect of the order $\rho$ of the generalized fractional BSEs in the diffusion processes.

In [12], Yavuz and Ozdemir proposed a novel definition of the Adomian decomposition method (ADM) to get an accurate and quick solution of T-FBSEs and generalized fractional BSEs utilizing initial condition. They obtained an approximate analytical solution to these equations.

Moreover, Ahmed and Elbadri [13] carried out the Sumudu decomposition method (SDM) coupled with the Adomian decomposition method to solve the fractional Newell-Whitehead-Segal equations (F-NWSEs). From their numerical results, they concluded that the proposed technique is straightforward to enforce and provide exact results. 
For solving the Newell-Whitehead-Segal equations [14], the authors presented two alternative exponential finite difference methods: implicit exponential finite difference scheme and fully implicit exponential finite difference scheme. Both techniques are demonstrated to be consistent. Furthermore, the local truncation errors are accurate to the first order in time and the second order in space. The approaches described are more accurate than other methods because they are computationally consistent, stable, and convergent.

Yavuz et al. constructed the conformable ADM and HPM, using the aforementioned conformable derivatives to solve various F-DEs such as diffusion equation, Black-Scholes equation (BSEs), and cable equation $[15,16]$. In [12], the authors proposed a novel definition of ADM to get an accurate and quick solution of the Black-Scholes equation of time-fractional order (F-BSEs) and the generalized Black-Scholes equation of fractional order (G-FBSEs) utilizing initial condition.

The main purpose of our study is to construct the Laplace transform homotopy perturbation method (LTHPM) for F-DEs using conformable derivatives.

The article is outlined as follows: some fundamental notions of conformable derivatives are revisited in Section 2. Subsequently, HPM is constructed using con-LT in Section 3. Finally, we apply in Section 4 the algorithm which is important to partial derivative equations (F-PDEs). The conclusion of this work is discussed in Section 5.

\section{Basic Notions}

This section presents some essential concepts of conformable derivatives, which will be necessary in our investigation.

Definition 1. Given $f:[0, \infty) \longrightarrow R$ as a function. Then, the $\alpha^{\text {th }}$ order conformable derivatives [1] is expressed as

$$
\left(T_{\alpha} f\right)(t)=\lim _{\varepsilon \longrightarrow 0} \frac{f\left(t+\varepsilon t^{1-\alpha}\right)-f(t)}{\varepsilon},
$$

$\forall t>0, \alpha \in(0,1]$. If $f$ is $\alpha$-differentiable ( $\alpha$-DifF) in some $(0, a), a>0$, and $\left(T_{\alpha} f\right)(t)$ exists, then it is expressed as

$$
\left(T_{\alpha} f\right)(0)=\lim _{t \longrightarrow 0^{+}}\left(T_{\alpha} f\right)(t) .
$$

Theorem 1 (See [1]). If a function $f:[0, \infty) \longrightarrow R$ is $\alpha$-DifF at $t_{0}>0, \alpha \in(0,1]$, then $f$ is continuous (ContF) at $t_{0}$.

Theorem 2 (See [1]). Suppose that $\alpha \in(0,1]$, and $f, h$ are $\alpha$-Diffs at a point $t>0$. Then, we get
(i) $T_{\alpha}(m f+w h)=m\left(T_{\alpha} f\right)+w\left(T_{\alpha} h\right), \forall m, w \in R$
(ii) $T_{\alpha}\left(t^{v}\right)=v t^{v-\alpha}, \forall v \in R$
(iii) $T_{\alpha}(\vartheta)=0, \forall$ constant function $f(t)=\vartheta$
(iv) $T_{\alpha}(f h)=f\left(T_{\alpha} h\right)+h\left(T_{\alpha} f\right)$
(v) $T_{\alpha}(f / h)=\left(\left(h\left(T_{\alpha} f\right)-f\left(T_{\alpha} h\right)\right) / h^{2}\right)$
(vi) If $f$ is DifF, then $\left(T_{\alpha} f\right)(t)=t^{1-\alpha}(d f / d t)(t)$

From the above definition, the conformable derivatives of some functions are expressed as

(i) $T_{\alpha}(1)=0$

(ii) $T_{\alpha}(\sin (a t))=a t^{1-\alpha} \cos (a t)$

(iii) $T_{\alpha}(\cos (a t))=a t^{1-\alpha} \sin (a t)$

(iv) $T_{\alpha}\left(e^{a t}\right)=a e^{a t}, a \in R$

In addition, some helpful formulas are expressed as

(i) $T_{\alpha}\left((1 / \alpha) t^{\alpha}\right)=1$

(ii) $T_{\alpha}\left(e^{(1 / \alpha) t^{\alpha}}\right)=e^{(1 / \alpha) t^{\alpha}}$

(iii) $T_{\alpha}\left(\sin \left((1 / \alpha) t^{\alpha}\right)\right)=\cos \left((1 / \alpha) t^{\alpha}\right)$

(iv) $T_{\alpha}\left(\cos \left((1 / \alpha) t^{\alpha}\right)\right)=-\sin \left((1 / \alpha) t^{\alpha}\right)$

Definition 2. The left-conformable derivative beginning from $a$ of function $f:[a, \infty) \longrightarrow R$ of order $\alpha \in(0,1]$ is expressed as [2]

$$
\left(T_{\alpha}^{a} f\right)(t)=\lim _{\varepsilon \longrightarrow 0} \frac{f\left(t+\varepsilon(t-a)^{1-\alpha}\right)-f(t)}{\varepsilon},
$$

when $a=0$, we have $\left(T_{\alpha} f\right)(t)$. If $f$ is $\alpha$-DifF in some $(a, b)$, then we get

$$
\left(T_{\alpha}^{a} f\right)(a)=\lim _{t \longrightarrow a^{+}}\left(T_{\alpha}^{a} f\right)(t)
$$

Theorem 3 (Chain Rule) [2]. Suppose that $f, h:(a, \infty) \longrightarrow R$ be left $\alpha$-DifFs, where $\alpha \in(0,1]$. Assume that $s(t)=f(h(t))$. $s(t)$ is $\alpha$ - Dif $F \forall t \neq a$ and $h(t) \neq 0$. Thus, we obtain

$$
\left(T_{\alpha}^{a} s\right)(t)=\left(T_{\alpha}^{a} f\right)(h(t)) \cdot\left(T_{\alpha}^{a} h\right)(t) .
$$

$$
\begin{aligned}
& \text { If } t=a \text {, then } \\
& \left(T_{\alpha}^{a} s\right)(a)=\lim _{t \longrightarrow a^{+}}\left(T_{\alpha}^{a} f\right)(h(t)) \cdot\left(T_{\alpha}^{a} h\right)(t) \cdot(h(t))^{\alpha-1} .
\end{aligned}
$$

Theorem 4 (See [2]). Supposed that $f$ is infinitely $\alpha$-DifF, for some $\alpha \in(0,1]$ at a neighborhood of a point $t_{0}$. Then, $f$ has a conformable power series expansion as

$$
f(t)=\sum_{k=0}^{\infty} \frac{\left((k) T_{\alpha}^{t_{0}}\right)\left(t_{0}\right)}{\alpha^{k} k !}\left(t-t_{0}\right)^{k \alpha}, \quad t_{0}<t<t_{0}+R^{(1 / \alpha)} .
$$

Hence, $\left((k) T_{\alpha}^{t_{0}}\right)\left(t_{0}\right)$ implies the application of conformable derivatives $k$ times.

The $\alpha$-conformable integral of a function $f$ is beginning from $a \geq 0$.

Definition 3 (See [1]). $I_{\alpha}^{a}(f)(t)=\int_{a}^{t}\left(f(x) / x^{1-\alpha}\right) \cdot \mathrm{d} x$, where this is a usual Riemann improper integral, $\alpha \in(0,1]$.

As a result, we have

Theorem 5. $T_{\alpha}^{a} I_{\alpha}^{a}(f)(t)=f(t)$, for $t \geq a$, where $f$ is any ContF in $I_{\alpha}^{\prime} s$ domain. 
Lemma 1. Suppose that $f:(a, b) \longrightarrow R$ is DifF and $\alpha \in(0,1]$. Then, $\forall a>0$, we get [2]

$$
I_{\alpha}^{a} T_{\alpha}^{a}(f)(t)=f(t)-f(a) .
$$

Finally, we recall the fractional Laplace transform (F-LT) [2]. In [3], a sufficient condition was accomplished to solve constant coefficient F-DEs via the con-LT method.

Definition 4. Suppose that $\alpha \in(0,1]$ and $f:[0, \infty) \longrightarrow R$ are real valued functions. Then, the F-LT of order $\alpha$ beginning from 0 of $f$ is expressed as

$$
L_{\alpha}[f(t)](s)=\int_{0}^{\infty} e^{-s\left(t^{\alpha} / \alpha\right)} f(t) \frac{\mathrm{d} t}{t^{1-\alpha}}
$$

Remark 1. The F-LT for the conformable derivatives is expressed as

$$
L_{\alpha}\left[\left(T_{\alpha} f\right)(t)\right](s)=s L_{\alpha}[f(t)](s)-f(0) .
$$

Thus, we have

Theorem 6. If $F_{\alpha}(s)=L_{\alpha}[f(t)](s)$ exists for $s>0$, then

(i) If $c$ a constant, then

$$
L_{\alpha}[c](s)=\frac{c}{s}
$$

(ii) Let $q$ be a constant:

$$
L_{\alpha}\left[t^{q}\right](s)=\alpha^{(q / \alpha)} \frac{\Gamma(1+(q / \alpha))}{s^{1+(q / \alpha)}} .
$$

(iii) If $c$ and $q$ are arbitrary constants,

$$
L_{\alpha}\left[t^{q} e^{c\left(t^{\alpha} / \alpha\right)}\right](s)=\alpha^{(q / \alpha)} \frac{\Gamma(1+(q / \alpha))}{(s-c)^{1+(q / \alpha)}}
$$

Definition 5. A function $f$ is considered as a conformable exponentially bounded if it satisfies $|f(t)| \leq M e^{c\left(t^{\alpha} / \alpha\right)}$, where $M$ and $c$ are the positive real constants and $\alpha \in(0,1], \forall$ sufficiently large $t$.

\section{Conformable Laplace Transform HPM}

A conformable version of HPM using LT has been introduced by Madani and Fathizadeh in [17]. In their research work, these authors discuss the effectiveness and ease of this technique to solve ordinary or partial differential equations (PDEs). In our case, a con-PDEs is given as follows:

$$
M_{\alpha} u(x, t)+N u(x, t)+R u(x, t)=g(x, t),
$$

with the initial condition (I.C):

$$
u(x, 0)=f(x)
$$

where $u$ is the two variables function, $M_{\alpha}=\left(\partial^{\alpha} / \partial t^{\alpha}\right)$ is a linear operator (LO) with conformable derivative of order $\alpha \in(0,1], R$ is the LO's other part, $N$ is a nonlinear operator (NLO), and $g(x, t)$ is a nonhomogeneous term. By solving for $M_{\alpha} u(x, t)$, equation (14) can be written as

$$
M_{\alpha} u(x, t)=g-N u-R u .
$$

By applying the con-LT to equation (16), we obtain

$$
L_{\alpha}\left[M_{\alpha} u(x, t)\right]=L_{\alpha}[g-N u-R u] .
$$

From Remark 1, the above equations can be rewritten as

$$
s L_{\alpha}[u(x, t)](s)-u(x, 0)=L_{\alpha}[g-N u-R u] .
$$

By substituting I.C, equation (18) can be written as

$$
L_{\alpha}[u(x, t)](s)=\frac{f(x)}{s}+\frac{1}{s} L_{\alpha}[g]-\frac{1}{s} L_{\alpha}[N u]-\frac{1}{s} L_{\alpha}[R u] .
$$

Furthermore, by applying inverse con-LT to equation (19), we get

$$
u(x, t)=G(x, t)-L_{\alpha}^{-1}\left[\frac{1}{s} L_{\alpha}[N u(x, t)+R u(x, t)]\right],
$$

where the term has been arisen from the source term, and the prescribed I.C is denoted by $G(x, t)$.

The HPM suggests the solution $u(x, t)$ to be decomposed into the infinite series of components $[18,19]$ :

$$
u(x, t)=\sum_{n=0}^{\infty} p^{n} u_{n}(x, t)
$$

and nonlinear term $N u(x, t)$ is decomposed to

$$
N u(x, t)=\sum_{n=0}^{\infty} p^{n} H_{n}(u)
$$

for some He's polynomials $H_{n}(u)[17,20]$ which are given by

$$
H_{n}\left(u_{0}, u_{1}, \ldots, u_{n}\right)=\frac{1}{n !} \frac{\partial^{n}}{\partial p^{n}}\left[N\left(\sum_{i=0}^{\infty} p^{i} u_{i}\right)\right], \quad n=0,1,2,3, \ldots
$$

By the substitution of equations (21) and (22) into equation (20), we have

$$
\sum_{n=0}^{\infty} p^{n} u_{n}(x, t)=G(x, t)-p\left(L_{\alpha}^{-1}\left[\frac{1}{s} L_{\alpha}\left[R \sum_{n=0}^{\infty} p^{n} u_{n}(x, t)+\sum_{n=0}^{\infty} p^{n} H_{n}(u)\right]\right]\right)
$$


which is the coupled con-FLT and HPM via He's polynomials. The approximation can be easily obtained by a comparison of all coefficients like powers of $p$ as follows:

$$
\begin{gathered}
p^{0}: u_{0}(x, t)=G(x, t), \\
p^{1}: u_{1}(x, t)=-L_{\alpha}^{-1}\left[\frac{1}{s} L_{\alpha}\left[R u_{0}(x, t)+H_{0}(u)\right],\right. \\
p^{2}: u_{2}(x, t)=-L_{\alpha}^{-1}\left[\frac{1}{s} L_{\alpha}\left[R u_{1}(x, t)+H_{1}(u)\right]\right], \\
p^{3}: u_{1}(x, t)=-L_{\alpha}^{-1}\left[\frac{1}{s} L_{\alpha}\left[R u_{2}(x, t)+H_{2}(u)\right]\right], \\
\vdots
\end{gathered}
$$

\section{Illustrative Examples}

The effectiveness of the con-LTHPM is shown in this section through four different problems: the time-fractional nonlinear homogeneous gas dynamics equation (F-GDEs), the time-fractional linear Newell-Whitehead-Segel equation (FNWSEs), the time-fractional diffusion-convection equation (F-DCEs), and the time-fractional linear Black-Scholes option pricing equation.

Example 1. Consider F-GDEs as follows:

$$
\frac{\partial^{\alpha} v(x, t)}{\partial t^{\alpha}}+v(x, t) \frac{\partial v(x, t)}{\partial x}-v(x, t)(1-v(x, t))=0, \quad t \geq 0,0 \leq x \leq 1,0<\alpha \leq 1,
$$

with I.C: $v(x, 0)=a e^{-x}$. If $\alpha=1$, then equation (26) becomes the classical nonlinear homogeneous GDEs [21].

Remark 2. Note that the mathematical expression of the classical GDEs is based on the physical laws of conservation.

By taking con-LT on equation (26) both sides and from the properties of con-LT, equation (26) reduces to

$$
L_{\alpha}[v(x, t)](s)=\frac{v(x, 0)}{s}+\frac{1}{s} L_{\alpha}\left[v-v^{2}-v \frac{\partial v}{\partial x}\right] .
$$

Using I.C and inverse con-LT, equation (27) reduces to

$$
v(x, t)=a e^{-x}+L_{\alpha}^{-1}\left[\frac{1}{s} L_{\alpha}\left[v-v^{2}-v \frac{\partial v}{\partial x}\right]\right] .
$$

The HPM is applied to obtain

$$
\sum_{n=0}^{\infty} p^{n} v_{n}(x, t)=a e^{-x}+p\left(L_{\alpha}^{-1}\left[\frac{1}{s} L_{\alpha}\left[\sum_{n=0}^{\infty} p^{n} v_{n}(x, t)-\sum_{n=0}^{\infty} p^{n} H_{n}(v)\right]\right]\right)
$$

where $H_{n}(v)$ represents He's polynomials.

The $1^{\text {st }}$ three parts of $H_{n}(v)$ are

$$
\begin{aligned}
& H_{0}(v)=v_{0}^{2}+v_{0} \frac{\partial v_{0}}{\partial x}, \\
& H_{1}(v)=2 v_{0} v_{1}+v_{0} \frac{\partial v_{1}}{\partial x}+v_{1} \frac{\partial v_{0}}{\partial x}, \\
& H_{2}(v)=2 v_{0} v_{2}+v_{0} \frac{\partial v_{2}}{\partial x}+v_{1} \frac{\partial v_{1}}{\partial x}+v_{2} \frac{\partial v_{0}}{\partial x}+v_{1}^{2},
\end{aligned}
$$


and so on.

The coefficient of power of $p$ is equated in equation (33) to get

$$
\begin{aligned}
& p^{0}: v_{0}(x, t)=a e^{-x} \\
& p^{1}: v_{1}(x, t)=L_{\alpha}^{-1}\left[\frac{1}{s} L_{\alpha}\left[v_{0}-H_{0}(v)\right]\right]=L_{\alpha}^{-1}\left[\frac{1}{s} L_{\alpha}\left[v_{0}-v_{0}^{2}-v_{0} \frac{\partial v_{0}}{\partial x}\right]\right]=a e^{-x} \frac{t^{\alpha}}{\alpha} \\
& p^{2}: v_{2}(x, t)=L_{\alpha}^{-1}\left[\frac{1}{s} L_{\alpha}\left[v_{1}-H_{1}(v)\right]\right]=L_{\alpha}^{-1}\left[\frac{1}{s} L_{\alpha}\left[v_{1}-2 v_{0} v_{1}-v_{0} \frac{\partial v_{1}}{\partial x}-v_{1} \frac{\partial v_{0}}{\partial x}\right]\right]=a e^{-x} \frac{1}{2 !}\left(\frac{t^{\alpha}}{\alpha}\right)^{2} \\
& p^{3}: v_{3}(x, t)=L_{\alpha}^{-1}\left[\frac{1}{s} L_{\alpha}\left[v_{2}-H_{2}(v)\right]\right]=L_{\alpha}^{-1}\left[\frac{1}{s} L_{\alpha}\left[v_{1}-2 v_{0} v_{2}-v_{0} \frac{\partial v_{2}}{\partial x}-v_{1} \frac{\partial v_{1}}{\partial x}-v_{2} \frac{\partial v_{0}}{\partial x}+v_{1}^{2}\right]\right]=a e^{-x} \frac{1}{3 !}\left(\frac{t^{\alpha}}{\alpha}\right)^{3} .
\end{aligned}
$$

Similarly, the approximations can further be obtained as follows:

$$
\begin{aligned}
& p^{4}: v_{4}(x, t)=a e^{-x} \frac{1}{4 !}\left(\frac{t^{\alpha}}{\alpha}\right)^{4}, \\
& p^{5}: v_{5}(x, t)=a e^{-x} \frac{1}{5 !}\left(\frac{t^{\alpha}}{\alpha}\right)^{5},
\end{aligned}
$$

$$
\begin{aligned}
v(x, t) & =v_{0}(x, t)+v_{1}(x, t)+v_{2}(x, t)+v_{3}(x, t)+\cdots \\
& =a\left(e^{-x}+e^{-x} \frac{t^{\alpha}}{\alpha}+e^{-x} \frac{1}{2 !}\left(\frac{t^{\alpha}}{\alpha}\right)^{2}+e^{-x} \frac{1}{3 !}\left(\frac{t^{\alpha}}{\alpha}\right)^{3}+e^{-x} \frac{1}{4 !}\left(\frac{t^{\alpha}}{\alpha}\right)^{4}+\cdots\right) \\
& =a e^{-x}\left(1+\frac{t^{\alpha}}{\alpha}+\frac{1}{2 !}\left(\frac{t^{\alpha}}{\alpha}\right)^{2}+\frac{1}{3 !}\left(\frac{t^{\alpha}}{\alpha}\right)^{3}+\frac{1}{4 !}\left(\frac{t^{\alpha}}{\alpha}\right)^{4}+\frac{1}{5 !}\left(\frac{t^{\alpha}}{\alpha}\right)^{5}+\cdots\right) \\
& =a e^{\left(t^{\alpha} / \alpha\right)-x} .
\end{aligned}
$$

The equation (26) exact solution with I.C: $v(x, 0)=a e^{-x}$ for $\alpha=1$ as a special case is found as follows:

$$
v(x, t)=a e^{t-x},
$$

which is the same solution in [21]. This result is verified graphically in Figure 1.

In Figure 2, the con-LTHPM's solution for various values of $\alpha$, i.e., $\alpha=0.7,0.9$ is represented. The F-GDEs is considered here with parameter $a=1$.

Example 2. Consider F-NWSEs as follows:

$$
\frac{\partial^{\alpha} v(x, t)}{\partial t^{\alpha}}=\frac{\partial^{2} v(x, t)}{\partial x^{2}}-3 v(x, t),
$$

with I.C: $v(x, 0)=e^{2 x}$. If $\alpha=1$, then equation (35) becomes an example of the classical linear NWSEs [22].

Remark 3. This equation has been applied in a wide variety of problems. For example, Faraday instability, nonlinear optics, Rayleigh-Bernard convection, or chemical reactions.

By taking con-LT on equation (45) both sides and from the properties of con-LT, equation (35) reduces to

$$
L_{\alpha}[v(x, t)](s)=\frac{v(x, 0)}{s+3}+\frac{1}{s+3} L_{\alpha}\left[\frac{\partial^{2} v(x, t)}{\partial x^{2}}\right]
$$

Using I.C and inverse con-LT, equation (36) reduces to 


$$
v(x, t)=e^{2 x-3\left(t^{\alpha} / \alpha\right)}+L_{\alpha}^{-1}\left[\frac{1}{s+3} L_{\alpha}\left[\frac{\partial^{2} v(x, t)}{\partial x^{2}}\right]\right] .
$$

The coefficients of the power of $p$ are compared as follows:

The HPM is applied to obtain

$$
\begin{aligned}
\sum_{n=0}^{\infty} p^{n} v_{n}(x, t)= & e^{2 x-3\left(t^{\alpha} / \alpha\right)} \\
& +p\left(L_{\alpha}^{-1}\left[\frac{1}{s+3} L_{\alpha}\left[\sum_{n=0}^{\infty} p^{n} \frac{\partial^{2} v_{n}(x, t)}{\partial x^{2}}\right]\right]\right)
\end{aligned}
$$

$$
\begin{aligned}
& p^{0}: v_{0}(x, t)=e^{2 x-3\left(t^{\alpha} / \alpha\right)} \\
& p^{1}: v_{1}(x, t)=L_{\alpha}^{-1}\left[\frac{1}{s+3} L_{\alpha}\left[\frac{\partial^{2} v_{0}(x, t)}{\partial x^{2}}\right]\right]=4 \frac{t^{\alpha}}{\alpha} e^{2 x-3\left(t^{\alpha} / \alpha\right)}, \\
& p^{2}: v_{2}(x, t)=L_{\alpha}^{-1}\left[\frac{1}{s+3} L_{\alpha}\left[\frac{\partial^{2} v_{1}(x, t)}{\partial x^{2}}\right]\right]=16 \frac{1}{2 !}\left(\frac{t^{\alpha}}{\alpha}\right)^{2} e^{2 x-3\left(t^{\alpha} / \alpha\right)}, \\
& p^{3}: v_{3}(x, t)=L_{\alpha}^{-1}\left[\frac{1}{s+3} L_{\alpha}\left[\frac{\partial^{2} v_{2}(x, t)}{\partial x^{2}}\right]\right]=64 \frac{1}{3 !}\left(\frac{t^{\alpha}}{\alpha}\right)^{3} e^{2 x-3\left(t^{\alpha} / \alpha\right)} .
\end{aligned}
$$

Similarly, approximations can further be obtained as follows:

$$
\begin{aligned}
& p^{4}: v_{4}(x, t)=256 \frac{1}{4 !}\left(\frac{t^{\alpha}}{\alpha}\right)^{4} e^{2 x-3\left(t^{\alpha} / \alpha\right)}, \\
& p^{5}: v_{5}(x, t)=512 \frac{1}{5 !}\left(\frac{t^{\alpha}}{\alpha}\right)^{5} e^{2 x-3\left(t^{\alpha} / \alpha\right)},
\end{aligned}
$$

$$
\begin{aligned}
v(x, t) & =v_{0}(x, t)+v_{1}(x, t)+v_{2}(x, t)+v_{3}(x, t)+\cdots \\
& =e^{2 x-3\left(t^{\alpha} / \alpha\right)}+e^{2 x-3\left(t^{\alpha} / \alpha\right)} \frac{4 t^{\alpha}}{\alpha}+e^{2 x-3\left(t^{\alpha} / \alpha\right)} \frac{1}{2 !}\left(\frac{4 t^{\alpha}}{\alpha}\right)^{2}+e^{2 x-3\left(t^{\alpha} / \alpha\right)} \frac{1}{3 !}\left(\frac{4 t^{\alpha}}{\alpha}\right)^{3}+e^{2 x-3\left(t^{\alpha} / \alpha\right)} \frac{1}{4 !}\left(\frac{4 t^{\alpha}}{\alpha}\right)^{4}+\cdots \\
& =e^{2 x-3\left(t^{\alpha} / \alpha\right)}\left(1+\frac{4 t^{\alpha}}{\alpha}+\frac{1}{2 !}\left(\frac{4 t^{\alpha}}{\alpha}\right)^{2}+\frac{1}{3 !}\left(\frac{4 t^{\alpha}}{\alpha}\right)^{3}+\frac{1}{4 !}\left(\frac{4 t^{\alpha}}{\alpha}\right)^{4}+\frac{1}{5 !}\left(\frac{4 t^{\alpha}}{\alpha}\right)^{5}+\cdots\right)=e^{2 x+3\left(t^{\alpha} / \alpha\right)}
\end{aligned}
$$

The equation (35) exact solution with $I v(x, 0)=e^{2 x}$, for $\alpha=1$ as a special case, is found as follows:

$$
v(x, t)=e^{2 x+t},
$$

and so on.

Substitute the above values into the following equation: 
Example 3. Consider F-DCEs as follows:

$$
\frac{\partial^{\alpha} v(x, t)}{\partial t^{\alpha}}=\frac{\partial^{2} v(x, t)}{\partial x^{2}}+\left(\cos x-\sin ^{2} x-1\right) v(x, t)
$$

with I.C: $v(x, 0)=(1 / 10) e^{\cos x-11}$. If $\alpha=1$, then equation (43) becomes an example of the classical DCEs [23].

Remark 4. The DCEs describe physical phenomena where particles, energy, or other physical quantities are transferred inside a physical system due to two processes: diffusion and convection.

By taking con-LT on equation (43) both sides and from the properties of con-LT, equation (43) reduces to

$$
\begin{aligned}
L_{\alpha}[v(x, t)](s)= & \frac{v(x, 0)}{s} \\
& +\frac{1}{s} L_{\alpha}\left[\frac{\partial^{2} v(x, t)}{\partial x^{2}}+\left(\cos x-\sin ^{2} x-1\right) v(x, t)\right] .
\end{aligned}
$$

Using I.C and inverse con-LT, equation (44) reduces to

$$
\begin{aligned}
v(x, t)= & \frac{1}{10} e^{\cos x-11} \\
& +L_{\alpha}^{-1}\left[\frac{1}{s} L_{\alpha}\left[\frac{\partial^{2} v(x, t)}{\partial x^{2}}+\left(\cos x-\sin ^{2} x-1\right) v(x, t)\right]\right] .
\end{aligned}
$$

The HPM is applied to obtain

$$
\sum_{n=0}^{\infty} p^{n} v_{n}(x, t)=\frac{1}{10} e^{\cos x-11}+p\left(L_{\alpha}^{-1}\left[\frac{1}{s} L_{\alpha}\left[\sum_{n=0}^{\infty} p^{n} \frac{\partial^{2} v_{n}(x, t)}{\partial x^{2}}+\left(\cos x-\sin ^{2} x-1\right) \sum_{n=0}^{\infty} p^{n} v_{n}(x, t)\right]\right]\right)
$$

The coefficients of the power of $p$ are compared here as follows:

$$
\begin{aligned}
& p^{0}: v_{0}(x, t)=\frac{1}{10} e^{\cos x-11}, \\
& p^{1}: v_{1}(x, t)=L_{\alpha}^{-1}\left[\frac{1}{s} L_{\alpha}\left[\frac{\partial^{2} v_{0}(x, t)}{\partial x^{2}}+\left(\cos x-\sin ^{2} x-1\right) v_{0}(x, t)\right]\right]=-\frac{1}{10} e^{\cos x-11} \frac{t^{\alpha}}{\alpha}, \\
& p^{2}: v_{2}(x, t)=L_{\alpha}^{-1}\left[\frac{1}{s} L_{\alpha}\left[\frac{\partial^{2} v_{1}(x, t)}{\partial x^{2}}+\left(\cos x-\sin ^{2} x-1\right) v_{1}(x, t)\right]\right]=\frac{1}{10} e^{\cos x-11} \frac{1}{2 !}\left(\frac{t^{\alpha}}{\alpha}\right)^{2}, \\
& p^{3}: v_{3}(x, t)=L_{\alpha}^{-1}\left[\frac{1}{s} L_{\alpha}\left[\frac{\partial^{2} v_{2}(x, t)}{\partial x^{2}}+\left(\cos x-\sin ^{2} x-1\right) v_{2}(x, t)\right]\right]=-\frac{1}{10} e^{\cos x-11} \frac{1}{3 !}\left(\frac{t^{\alpha}}{\alpha}\right)^{3} .
\end{aligned}
$$




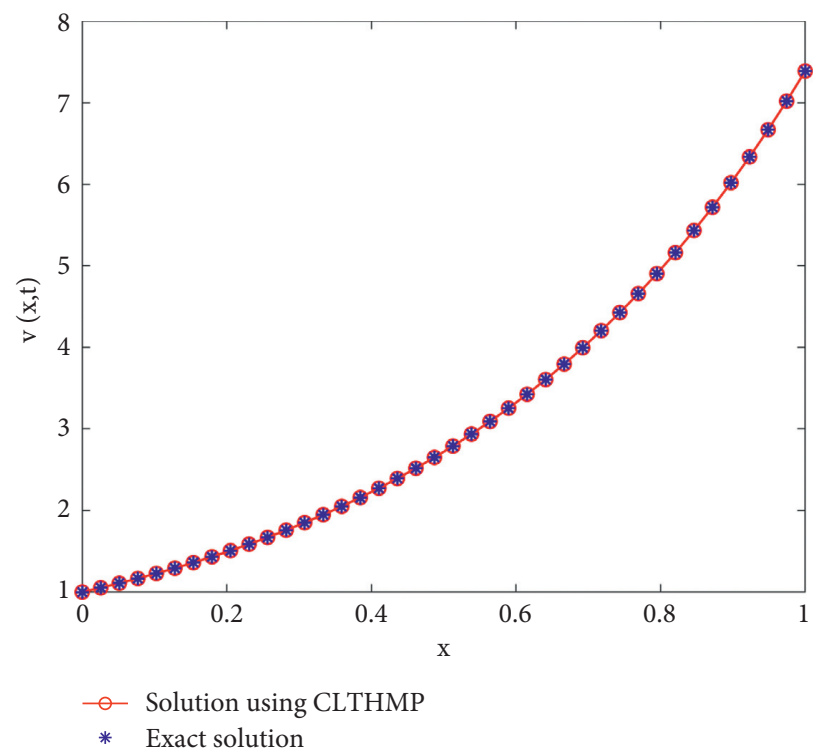

Figure 1: Comparison of the numerical solution and exact solution for $\alpha=1$.
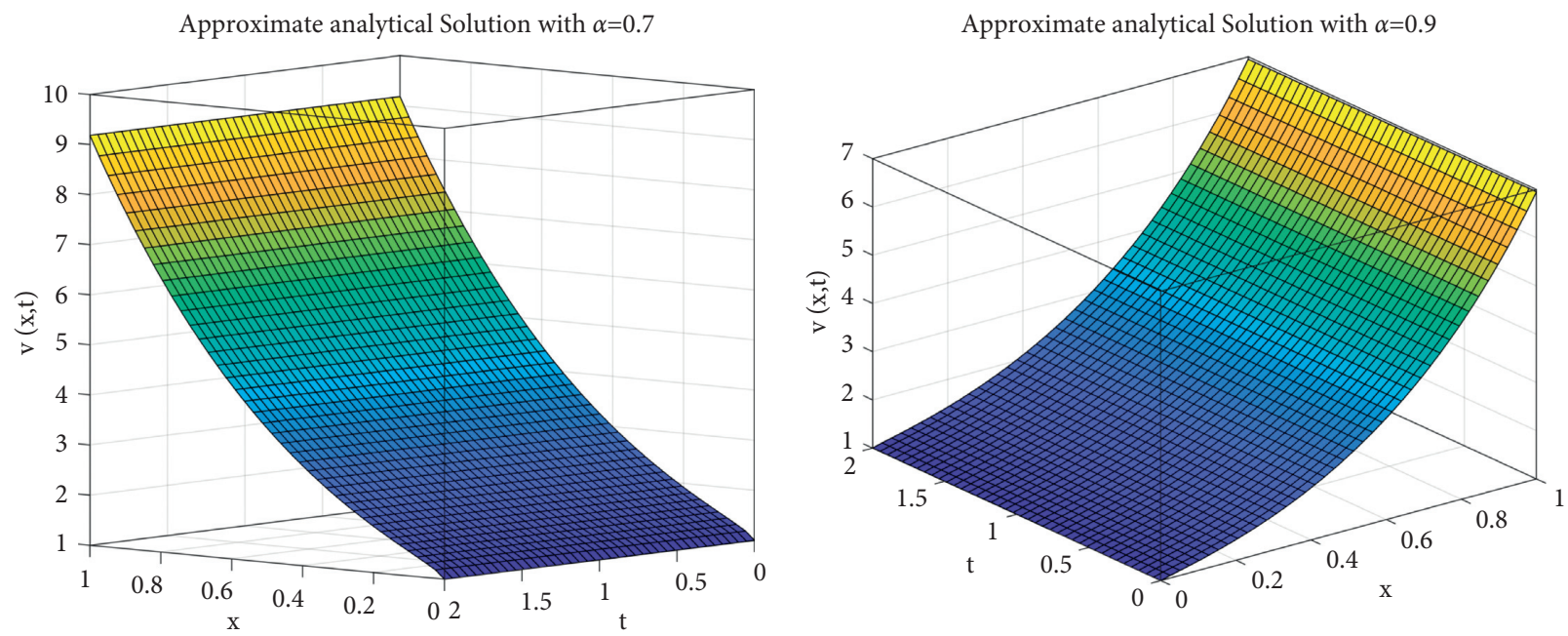

Figure 2: Con-LTHPM's solutions of the F-GDEs.

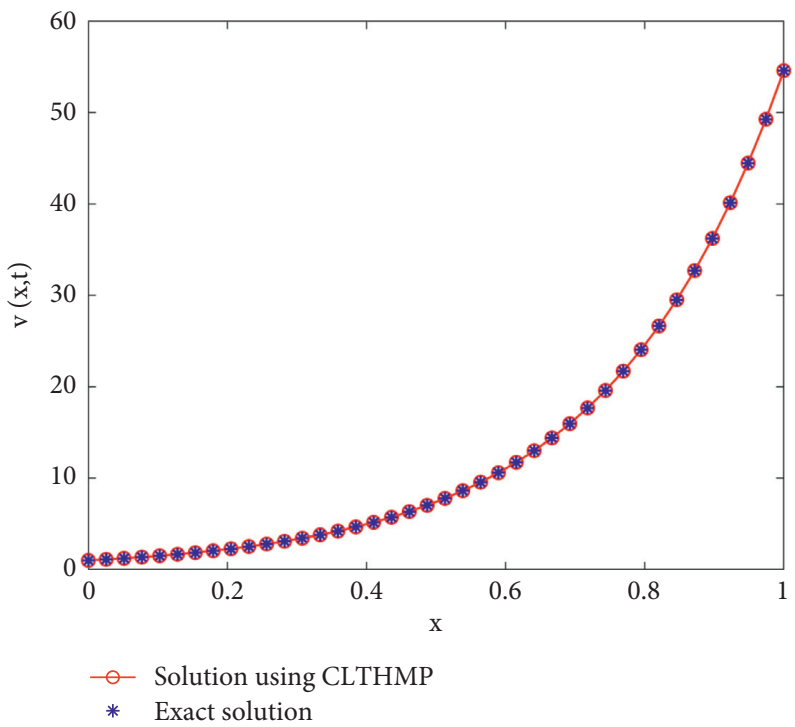

Figure 3: Comparison of the numerical solution and exact solution for $\alpha=1$. 
Similarly, approximations can further be obtained as follows:

$$
\begin{aligned}
& p^{4}: v_{4}(x, t)=\frac{1}{10} e^{\cos x-11} \frac{1}{4 !}\left(\frac{t^{\alpha}}{\alpha}\right)^{4}, \\
& p^{5}: v_{5}(x, t)=-\frac{1}{10} e^{\cos x-11} \frac{1}{5 !}\left(\frac{t^{\alpha}}{\alpha}\right)^{5},
\end{aligned}
$$

and so on.

Substitute the above values in the following equation:

$$
\begin{aligned}
v(x, t) & =v_{0}(x, t)+v_{1}(x, t)+v_{2}(x, t)+v_{3}(x, t)+\cdots \\
& =\frac{1}{10} e^{\cos x-11}\left(1-\frac{t^{\alpha}}{\alpha}+\frac{1}{2 !}\left(\frac{t^{\alpha}}{\alpha}\right)^{2}-\frac{1}{3 !}\left(\frac{t^{\alpha}}{\alpha}\right)^{3}+\frac{1}{4 !}\left(\frac{t^{\alpha}}{\alpha}\right)^{4}-\frac{1}{5 !}\left(\frac{t^{\alpha}}{\alpha}\right)^{5}+\cdots\right)=\frac{1}{10} e^{\cos x-11-\left(t^{\alpha} / \alpha\right)} .
\end{aligned}
$$

The equation (43) exact solution with I.C: $v(x, 0)=(1 / 10) e^{\cos x-11}$, for $\alpha=1$ as a special case, is found as follows:

$$
v(x, t)=\frac{1}{10} e^{\cos x-11-t}
$$

which is the same solution with [23]. This result is also verified graphically, as shown in Figure 5.

In Figure 6, the con-LTHPM's solution for various values of $\alpha$, i.e., $\alpha=0.7,0.9$ is represented.

Example 4. Consider the F-BSEs:

$$
\frac{\partial^{\alpha} v(x, t)}{\partial t^{\alpha}}=\frac{\partial^{2} v(x, t)}{\partial x^{2}}+(k-1) \frac{\partial v(x, t)}{\partial x}-k v(x, t), \quad t>0, x \in R, 0<\alpha \leq 1
$$

with I.C: $v(x, 0)=\max \left\{e^{x}-1,0\right\}$. If $\alpha=1$, then equation (51) becomes the classical linear BSEs [24].

Remark 5. Equation (51) contains only two dimensionless parameters $k=\left(2 r / \sigma^{2}\right)$, where $k$ represents the balance between the rates of interest and the variability of the return on stocks and the dimensionless time to expiry $\left(\sigma^{2} T / 2\right)$.
By taking con-LT on equation (51) both sides and from the properties of con-LT, equation (51) reduces to

$$
L_{\alpha}[v(x, t)](s)=\frac{v(x, 0)}{s+k}+\frac{1}{s+k} L_{\alpha}\left[\frac{\partial^{2} v(x, t)}{\partial x^{2}}+(k-1) \frac{\partial v(x, t)}{\partial x}\right] .
$$

Using I.C and inverse con-LT, equation (52) reduces to

$$
v(x, t)=\max \left\{e^{x}-1,0\right\} e^{-k\left(t^{\alpha} / \alpha\right)}+L_{\alpha}^{-1}\left[\frac{1}{s+k} L_{\alpha}\left[\frac{\partial^{2} v(x, t)}{\partial x^{2}}+(k-1) \frac{\partial v(x, t)}{\partial x}\right]\right] .
$$

The HPM is applied to obtain

$$
\sum_{n=0}^{\infty} p^{n} v_{n}(x, t)=\max \left\{e^{x}-1,0\right\} e^{-k\left(t^{\alpha} / \alpha\right)}+p\left(L_{\alpha}^{-1}\left[\frac{1}{s+k} L_{\alpha}\left[\sum_{n=0}^{\infty} p^{n} \frac{\partial^{2} v_{n}(x, t)}{\partial x^{2}}+(k-1) \sum_{n=0}^{\infty} p^{n} \frac{\partial v_{n}(x, t)}{\partial x}\right]\right]\right)
$$



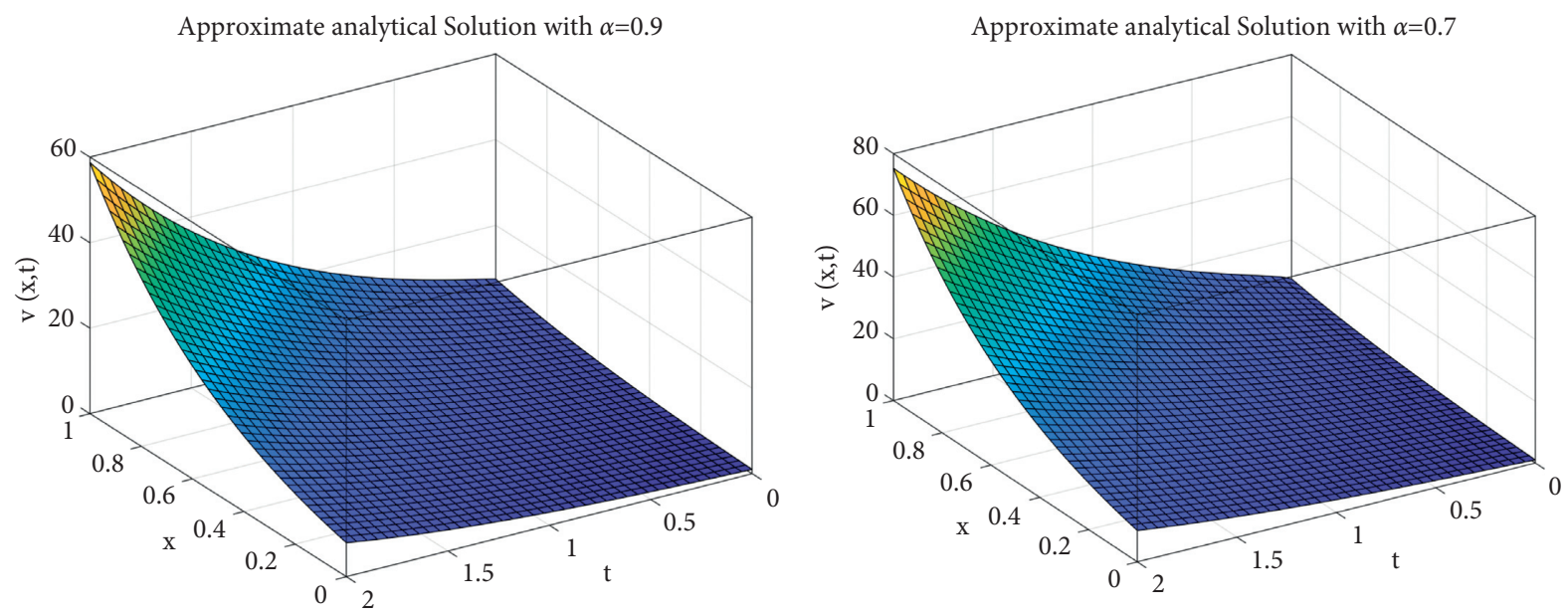

FIGURE 4: Con-LTHPM's solutions of the F-NWSEs.

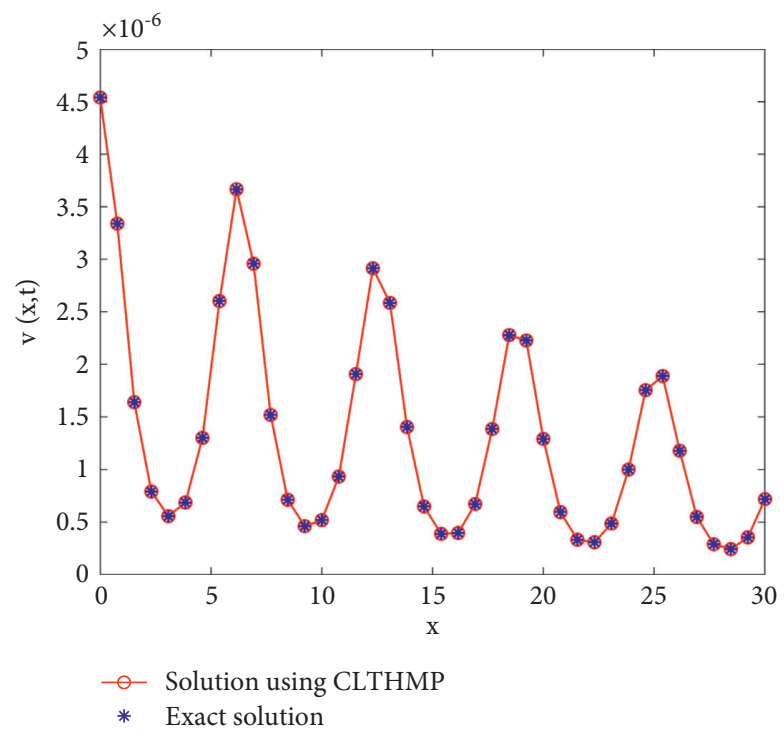

FiguRE 5: Comparison of the numerical solution and exact solution for $\alpha=1$.

The coefficients of the power of $p$ are compared to get

$$
\begin{aligned}
& p^{0}: v_{0}(x, t)=\max \left\{e^{x}-1,0\right\} e^{-k\left(t^{\alpha} / \alpha\right)}, \\
& p^{1}: v_{1}(x, t)=L_{\alpha}^{-1}\left[\frac{1}{s+k} L_{\alpha}\left[\frac{\partial^{2} v_{0}(x, t)}{\partial x^{2}}+(k-1) \frac{\partial v_{0}(x, t)}{\partial x}\right]\right]=\frac{k t^{\alpha}}{\alpha} e^{x-k\left(t^{\alpha} / \alpha\right)}, \\
& p^{2}: v_{2}(x, t)=L_{\alpha}^{-1}\left[\frac{1}{s+k} L_{\alpha}\left[\frac{\partial^{2} v_{1}(x, t)}{\partial x^{2}}+(k-1) \frac{\partial v_{1}(x, t)}{\partial x}\right]\right]=\frac{1}{2 !}\left(\frac{K t^{\alpha}}{\alpha}\right)^{2} e^{x-k\left(t^{\alpha} / \alpha\right)}, \\
& p^{3}: v_{3}(x, t)=L_{\alpha}^{-1}\left[\frac{1}{s+k} L_{\alpha}\left[\frac{\partial^{2} v_{2}(x, t)}{\partial x^{2}}+(k-1) \frac{\partial v_{2}(x, t)}{\partial x}\right]\right]=\frac{1}{3 !}\left(\frac{k t^{\alpha}}{\alpha}\right)^{3} e^{x-k\left(t^{\alpha} / \alpha\right)} .
\end{aligned}
$$



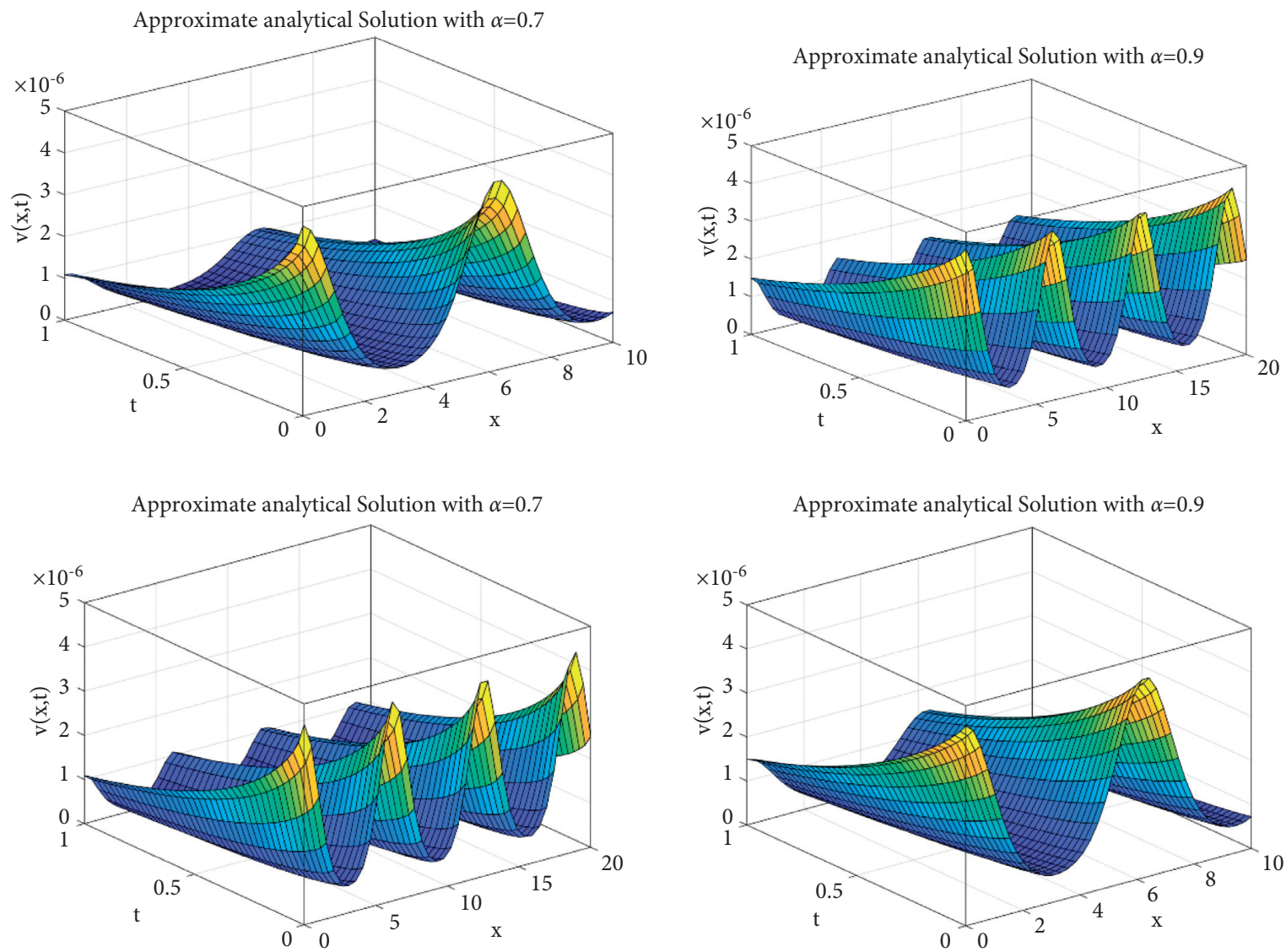

Figure 6: Con-LTHPM's solutions of the F-DCEs.

Similarly, approximations can further be obtained as follows:

$$
\begin{aligned}
& p^{4}: v_{4}(x, t)=\frac{1}{4 !}\left(\frac{k t^{\alpha}}{\alpha}\right)^{4} e^{x-k\left(t^{\alpha} / \alpha\right)} \\
& p^{5}: v_{5}(x, t)=\frac{1}{5 !}\left(\frac{k t^{\alpha}}{\alpha}\right)^{5} e^{x-k\left(t^{\alpha} / \alpha\right)}
\end{aligned}
$$

$$
\begin{aligned}
v(x, t) & =v_{0}(x, t)+v_{1}(x, t)+v_{2}(x, t)+v_{3}(x, t)+\cdots \\
& =\max \left\{e^{x}-1,0\right\} e^{-k\left(t^{\alpha} / \alpha\right)}+e^{x-k\left(t^{\alpha} / \alpha\right)}\left(\frac{k t^{\alpha}}{\alpha}+\frac{1}{2 !}\left(\frac{k t^{\alpha}}{\alpha}\right)^{2}+\frac{1}{3 !}\left(\frac{k t^{\alpha}}{\alpha}\right)^{3}+\frac{1}{4 !}\left(\frac{k t^{\alpha}}{\alpha}\right)^{4}+\cdots\right) \\
& =\max \left\{e^{x}-1,0\right\} e^{-k\left(t^{\alpha} / \alpha\right)}+e^{x}\left(1-e^{-k\left(t^{\alpha} / \alpha\right)}\right) .
\end{aligned}
$$



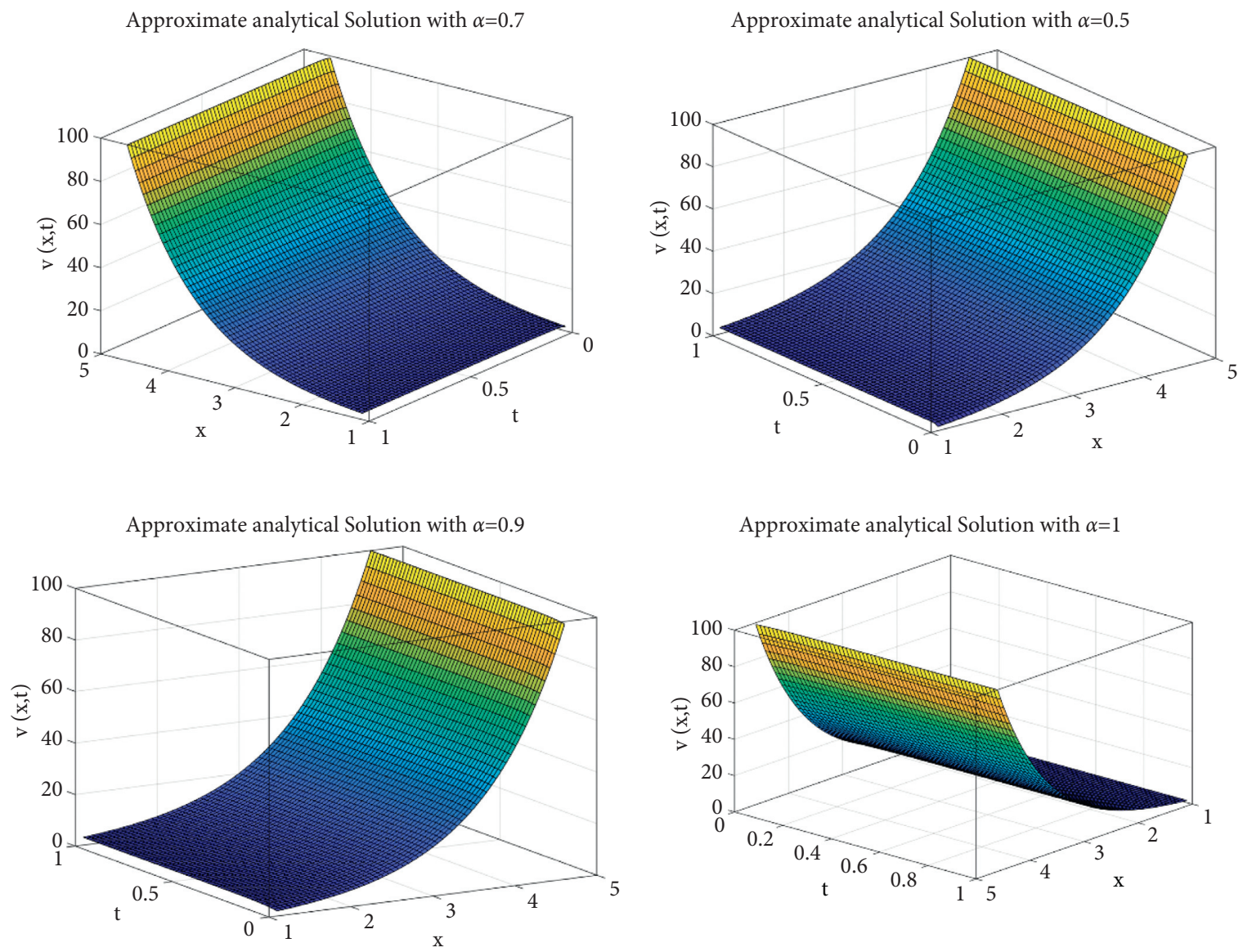

Figure 7: Con-LTHPM's solutions of the F-BSEs.

The equation (35) exact solution with I.C: $v(x, 0)=\left\{e^{x}-1,0\right\}$, for $\alpha=1$ as a special case, is found as follows:

$$
v(x, t)=\left\{e^{x}-1,0\right\} e^{-k t}+e^{x}\left(1-e^{-k t}\right),
$$

which is the same solution in [24].

In Figure 7, the con-LTHPM's solution for various values of $\alpha$, i.e., $\alpha=0.5,0.7,0.9,1$ is represented. In this example, F-BSEs is considered for $0<\alpha \leq 1$.

\section{Conclusion}

The approximate analytical solutions have been obtained in this work for time F-PDEs, using a numerical method based on the con derivatives, a concept widely used in the field of applications in recent years. We have constructed a con-version of the HPM via LT. This technique's efficiency and approximation have been verified through four important problems. From the illustrative examples, the results using this technique coincide the corresponding exact solution. As a result, our technique can be applicable to many initial-value problems and F-PDEs including linear and nonlinear ones. In fact, future research will focus on studying this conformable methodology of important classic problems such as the in-homogeneous fractional cable equation, the Burgers equation with fractional order of space and time, or the in-homogeneous nonlinear Klein-Gordon equation.

\section{Data Availability}

No data were used to support this study.

\section{Conflicts of Interest}

The authors declare that they have no conflicts of interest.

\section{Authors' Contributions}

Sajad Iqbal involved in actualization, methodology, formal analysis, validation, investigation, and initial draft. Mohammed K. A. Kaabar involved in actualization, methodology, formal analysis, validation, investigation, initial draft, and supervision of the original draft and editing. Francisco Martínez involved in actualization, validation, methodology, formal analysis, investigation, and initial draft. All authors read and approved the final version.

\section{References}

[1] A. Kilbas, H. Srivastava, and J. Trujillo, Theory and Applications of Fractional Differential Equations, North-Holland, Amsterdam, Netherlands, 2006.

[2] K. S. Miller, An Introduction to Fractional Calculus and Fractional Differential Equations, J. Wiley and Sons, Hoboken, NJ, USA, 1993. 
[3] R. Khalil, M. Al Horani, A. Yousef, and M. Sababheh, "A new definition of fractional derivative," Journal of Computational and Applied Mathematics, vol. 264, pp. 65-70, 2014.

[4] D. Zhao and M. Luo, "General conformable fractional derivative and its physical interpretation," Calcolo, vol. 54, no. 3 , pp. 903-917, 2017.

[5] R. Khalil, M. Al Horani, and M. Abu Hammad, "Geometric meaning of conformable derivative via fractional cords," Journal of Mathematics and Computer Science, vol. 19, no. 4, pp. 241-245, 2019.

[6] N. Bildik and H. Bayramoglu, "The solution of two dimensional nonlinear differential equations by the adomian decomposition method," Applied Mathematics and Computation, vol. 163, no. 2, pp. 519-524, 2005.

[7] N. Bildik, A. Konuralp, F. O. Bek, and S. Küçükarslan, "Solution of different type of the partial differential equation by differential transform method and adomian's decomposition method," Applied Mathematics and Computation, vol. 172, no. 1, pp. 551-567, 2006.

[8] M. Javidi and B. Ahmad, "Numerical solution of fractional partial differential equations by numerical laplace inversion technique," Advances in Difference Equations, vol. 2013, no. 1, 375 pages, 2013.

[9] M. Madani, M. Fathizadeh, Y. Khan, and A. Yildirim, "On the coupling of the homotopy perturbation method and laplace transformation," Mathematical and Computer Modelling, vol. 53, no. 9, pp. 1937-1945, 2011.

[10] S. J. Johnston, H. Jafari, S. P. Moshokoa, V. M. Ariyan, and D. Baleanu, "Laplace homotopy perturbation method for burgers equation with space-and time-fractional order," Open Physics, vol. 14, no. 1, pp. 247-252, 2016.

[11] A. N. Fall, S. N. Ndiaye, and N. Sene, "Black-scholes option pricing equations described by the caputo generalized fractional derivative," Chaos, Solitons \& Fractals, vol. 125, pp. 108-118, 2019.

[12] M. Yavuz and N. Özdemir, "A quantitative approach to fractional option pricing problems with decomposition series," Konuralp Journal of Mathematics, vol. 6, no. 1, pp. 102-109, 2018.

[13] S. A. Ahmed and M. Elbadri, "Solution of newell-whiteheadsegal equation of fractional order by using sumudu decomposition method," Mathematics and Statistics, vol. 8, no. 6, pp. 631-636, 2020.

[14] N. Hilal, S. Injrou, and R. Karroum, "Exponential finite difference methods for solving Newell-Whitehead-Segel equation," Arabian Journal of Mathematics, vol. 9, no. 2, pp. 367-379, 2020.

[15] M. Yavuz and N. Ozdemir, "A different approach to the European option pricing model with fractional operator," Mathematical Modelling of Natural Phenomena, vol. 13, pp. 1-12, 2018.

[16] M. Yavuz, "Novel solution methods for initial boundary value problems of fractional order with conformable differentiation," An International Journal of Optimization and Control, vol. 8, no. 1, pp. 1-7, 2018.

[17] M. Madani and M. Fathizadeh, "Homotopy perturbation algorithm using laplace transformation," Nonlinear Science Letters A, vol. 1, pp. 263-267, 2010.

[18] Y. Khan and Q. Wu, "Homotopy perturbation transform method for nonlinear equations using he's polynomials," Computers \& Mathematics with Applications, vol. 61, no. 8, pp. 1963-1967, 2011.
[19] S. T. Mohyud-Din and A. Yildirim, "Homotopy perturbation method for advection problems," Nonlinear Science Letters A, vol. 1, pp. 307-312, 2010.

[20] S. T. Mohyud-Din, M. A. Noor, and K. I. Noor, "Traveling wave solutions of seventh-order generalized KdV equation using he's polynomials," International Journal of Nonlinear Sciences and Numerical Simulation, vol. 10, no. 2, pp. 227-233, 2009.

[21] P. Kiran, G. Badhane, and V. H. Pradhan, "Application of Laplace transform homotopy perturbation method to gas dynamic equation: a modified approach," International Journal of Research in Engineering and Technology, vol. 5, no. 5, pp. 409-411, 2016.

[22] H. K. Jassim, "Homotopy perturbation algorithm using laplace transform for newell-witehead-segel equation," International Journal of Applied Mathematics and Mechanics, vol. 2, no. 4, pp. 8-12, 2015.

[23] S. Gupta, D. Kumar, and J. Singh, "Analytical solutions of convection-diffusion problems by combining laplace transform method and homotopy perturbation method," Alexandria Engineering Journal, vol. 54, no. 3, pp. 645-651, 2015.

[24] F. Black and M. Scholes, "The pricing of options and corporate liabilities," Journal of Political Economy, vol. 81, no. 3, pp. 637-654, 1973. 